\title{
Expression of Filaggrin in Normal and Keratinized Conjunctiva
}

\author{
Anne Sofie Kragegaard Lund ${ }^{1,3}$, Steffen Heegaard ${ }^{1,2}$, Jan Ulrik Prause ${ }^{1}$, Peter Bjerre Toft ${ }^{2}$ and \\ Lone $\mathrm{Skov}^{*}, 3$ \\ ${ }^{I}$ Institute of Neuroscience and Pharmacology, Eye Pathology Institute, University of Copenhagen, Copenhagen, \\ Denmark \\ ${ }^{2}$ Department of Ophthalmology, Glostrup Hospital, University of Copenhagen, Copenhagen, Denmark \\ ${ }^{3}$ Department of Dermato-allergology, Gentofte Hospital, University of Copenhagen, Denmark
}

\begin{abstract}
Purpose: We wanted to investigate filaggrin expression in normal conjunctiva and in conjunctiva exposed to different degrees of mechanical stress. Mechanical stress results in parakeratinization of the conjunctiva. If filaggrin is expressed in the milder forms of parakeratinization, it might be used as a sensitive marker of mechanical stress.

Methods: Immunohistochemical staining using antibodies to filaggrin was performed on paraffin sections of normal human conjunctiva, and on conjunctiva with different degrees of mechanical conjunctival stress.

Results: Filaggrin was not expressed in the normal conjunctiva, nor in conjunctiva with milder forms of mechanical stress. Intense staining of filaggrin was seen in the conjunctiva of a patient with Stevens-Johnson syndrome, and marked expression of filaggrin was found in the conjunctival epithelium of a patient with moderate dysplasia of the conjunctiva.

Conclusion: Filaggrin is not a sensitive marker of mechanical stress; it is, however, expressed in some moderate and severe forms of parakeratinization of the conjunctiva.
\end{abstract}

Keywords: Filaggrin, conjunctiva, parakeratinized, mechanical stress.

\section{INTRODUCTION}

Filaggrin is a barrier protein that is found in the outer keratinized layer of the epidermis. It is also expressed in parakeratinized areas of human oral epithelium [1] and the exocervix epithelium [2]. Previous studies have shown no or very weak expression of filaggrin in the human corneal epithelium $[3,4]$ and no or weak expression in the healthy human conjunctiva [4-6]. In one study, filaggrin was found to be expressed in severe ocular surface diseases such as alkali burns and Stevens-Johnson syndrome, at places where the epithelium had keratinized [6].

Long-term mechanical stress to the conjunctival epithelium leads to atrophy or metaplasia, in which there is loss of goblet cells, conversion from stratified columnar to stratified squamous configuration, and keratinization [7]. Until now, the expression of filaggrin has only been studied in normal human conjunctiva and in conjunctiva that is severely damaged and keratinized.

Our aim was to study the expression of filaggrin in condition with different degrees of mechanical stress and parakeratinization of the conjunctiva. To do this, we investigated the expression in normal conjunctiva, in the conjunctiva of patients with pinguecula, pterygium or in

*Address correspondence to this author at the Department of Dermatoallergology, Gentofte Hospital, Niels Andersens Vej 65, 2900 Hellerup, Denmark; Tel: +45 39773204; Fax: +45 39777615;

E-mail: lone.skov.02@regionh.dk moderate dysplasia of the conjunctiva as well as in StevensJohnson syndrome. Under these conditions thickening of the conjunctiva causes rubbing by the eyelids and mechanical stress. If filaggrin is expressed in milder forms of mechanical stress and parakeratinization, it might be used as a marker of mechanical stress in the conjunctiva.

\section{MATERIALS AND METHODS}

Formalin-fixed, paraffin-embedded blocks of five orbital exenterations including normal human eyes and eyelids from the same patients were used to investigate filaggrin expression in normal conjunctiva, and in skin as a positive control. We investigated tissue blocks from three middleaged patients (mean age 55 years) and two elderly patients (mean age 76 years). The orbital exenterations were performed due to cancer or Wegener's granulomatosis. The diseases did not involve the conjunctiva being investigated or the skin of the eyelid.

In addition, conjunctiva specimens from two patients with pterygium, two patients with pinguecula, one patient with moderate dysplasia of the conjunctiva due to human papilloma virus (HPV), and one patient with StevensJohnson syndrome were studied for filaggrin expression. All specimens were obtained from the Eye Pathology Institute, University of Copenhagen, Denmark.

The study was approved by the Danish Data Protection Agency and the local ethics committee (H-1-2011-154). 


\section{Immunohistochemistry}

Two $4.5 \mu \mathrm{m}$ sections per patient were cut from the paraffin-embedded specimens. The sections were deparaffinized, boiled for $20 \mathrm{~min}$ in TrisEDTA buffer $(\mathrm{pH}=9)$ for antigen retrieval, and incubated for $25 \mathrm{~min}$ with the primary antibody to filaggrin, diluted 1:50 (clone no. 15C10, code no. NCL-Filaggrin; Novocastra Laboratories Ltd, Newcastle upon Tyne, UK). Then the sections were incubated with a secondary antibody (kit code K5003; Dako $\mathrm{A} / \mathrm{S}$ ) for $25 \mathrm{~min}$ followed by a peroxidase blocking solution $\left(1 \% \mathrm{H}_{2} \mathrm{O}_{2}\right)$ for eight min and with a dilution of streptavidin peroxidase (kit code K5003; Dako A/S). Before and after each incubation with antibody or streptavidin peroxidase, the sections were washed with PBS. They were visualized using AEC chromogen (kit code K5003; Dako A/S). All sections, except for the conjunctiva from the patient with moderate dysplasia of the conjunctiva, were stained simultaneously to ensure that samples for comparison were treated in exactly the same way. Positive and negative controls were included to ensure good antigen retrieval and to prevent false positives.

\section{RESULTS}

\section{Expression of Filaggrin in Normal Human Conjunctiva}

No expression of filaggrin was seen in the healthy conjunctivas (Fig. 1F). Positive controls tissue, i.e. skin from the eyelids of the same patients, showed strong filaggrin expression in the outer, keratinized epithelium (Fig. 1G).

\section{Expression of Filaggrin in Abnormal Conjunctiva}

\section{Pinguecula}

The two patients with pinguecula were men aged 56 and 81 years. By staining, there was no evidence of filaggrin expression in the conjunctivas of these patients.

\section{Pterygium}

One patient with pterygium was a man aged 46 years and the other was a woman aged 64 years. We did not find any expression of filaggrin in the conjunctiva specimens of these two patients (Fig. 1A, B).

\section{Stevens-Johnson Syndrome}

We investigated three different samples from a male patient with Stevens-Johnson syndrome: one biopsy taken from the conjunctiva of the right eye and one taken from the conjunctiva of the left eye, when the patient was 23 years old. The third biopsy was from the left conjunctiva when the patient was 29 years old (Fig. 1C). Intense staining of filaggrin was seen in the superficial layers of all three samples (Fig. 1D).

\section{Moderate Dysplasia of the Conjunctiva}

The patient with moderate dysplasia was 70 years old at the time of resection. Marked expression of filaggrin was found in the parakeratinized areas of the conjunctiva (Fig. 1E).

\section{DISCUSSION}

Filaggrin is part of the normal skin barrier, where it plays an essential role in the regulation of epidermal homeostasis $[8,9]$. It is expressed in keratinocytes during the terminal differentiation, and is therefore involved in keratinization of the skin [10]. Several mutations in the gene that encodes filaggrin (FLG) have been identified, and linked to skin diseases $[11,12]$. Homozygosity results in no production of filaggrin. In the present study, normal conjunctiva from the control group did not express any filaggrin. This is consistent with results of previous studies [5,6]. To ensure that was not caused by a mutation in the filaggrin gene, we tested both conjunctiva and skin from the same patient at the same time. They all showed expression of filaggrin in the skin and no expression of filaggrin in the conjunctival epithelium, thereby eliminating the possibility that filaggrin was not detected in the conjunctiva because of a mutation in the gene that encodes the protein.

One study [4] found that paraformaldehyde destroyed the antigenicity of the filaggrin molecule in biopsy specimens of human skin. No information on the chemicals used to unmask the epitope was given, but since trypsin was used to pre-treat cytokeratins, it might have been used for filaggrin. We used a Tris-EDTA buffer, and there was a positive result for filaggrin in the controls.

One study has also shown that severe damage to the conjunctiva - in the form of Stevens-Johnson syndrome, ocular cicatricial pemphigoid, or chemical injuries in the chronic cicatricial phase - induces the upregulation of filaggrin [6].

In the present study, we found filaggrin expression in the conjunctival epithelium of a patient with moderate dysplasia of the conjunctiva. In addition, we found filaggrin expression in two specimens taken six years apart in the conjunctiva of a patient with Stevens-Johnson syndrome. This confirms that filaggrin is expressed when the conjunctiva becomes parakeratinized. Our findings also show that in severely parakeratinized conjunctiva, the expression of filaggrin may be stable over many years. Filaggrin was also expressed in conjunctival dysplasia, which is characterized by mild parakeratinization.

To our knowledge, filaggrin expression in conjunctivae with milder degrees of parakeratinization has not previously been described. Filaggrin was not detected in the conjunctiva of patients with pterygium and pinguecula. Our hypothesis was that even mild parakeratinization may lead to filaggrin expression. However, we found no filaggrin expression in patients suffering from pterygium or pinguecula. We did not have skin samples from these patients, and the lack of filaggrin expression might have been caused by a mutation in filaggrin gene. However, since only $9 \%$ of the population of European origin are carriers of the most common filaggrin mutations [13], it is unlikely that the two patients with pterygium and the two patients with pinguecula would all be homozygotes for a mutation. More patients would have to be tested to make sure that these results was not caused by a 

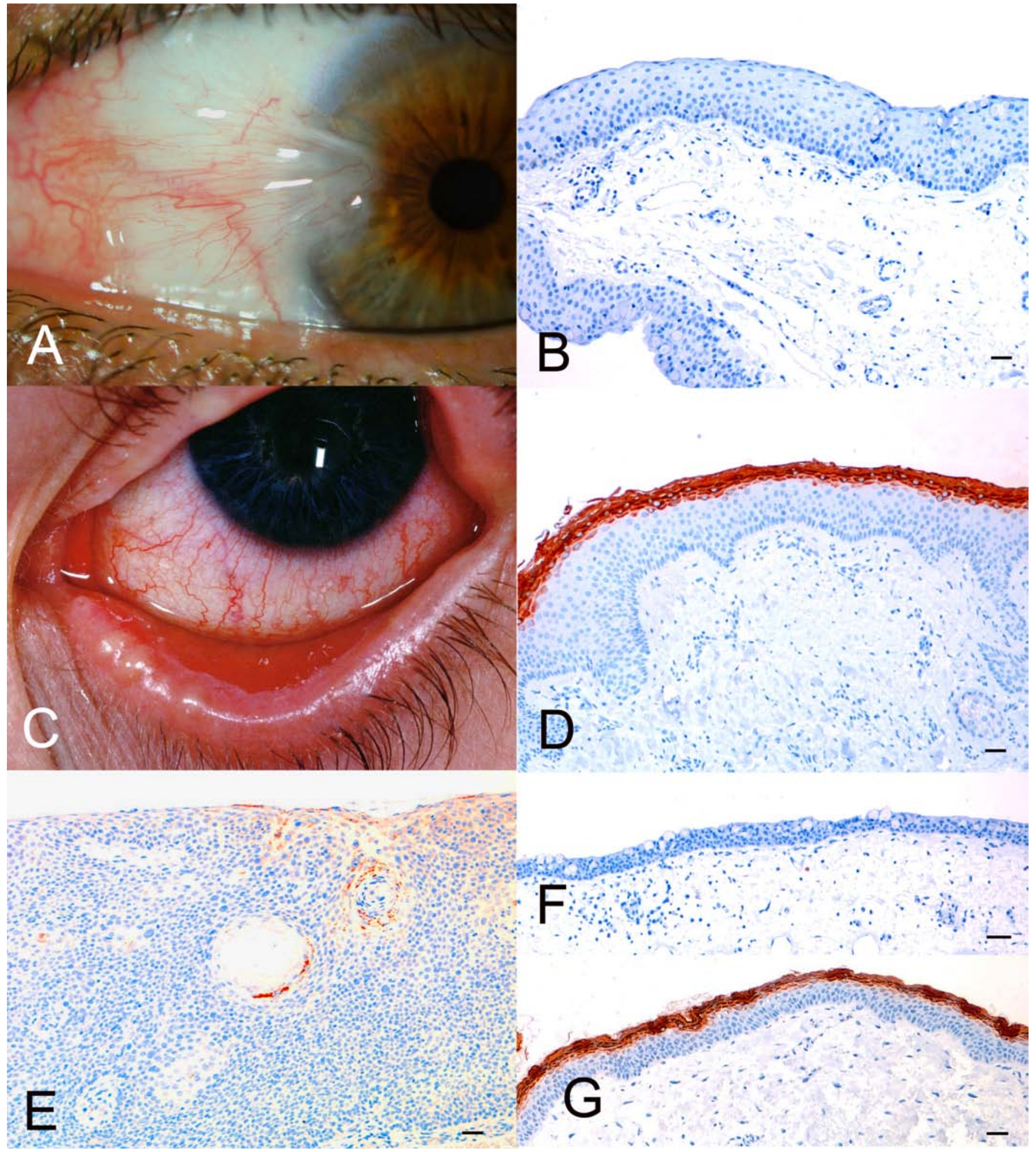

Fig. (1). A. Nasal pterygium. B. Micrograph of pterygium stained with anti-filaggrin. Bar $=100 \mu \mathrm{m}$. Note the complete lack of filaggrin expression. C. Late stage of Stevens-Johnson syndrome. D. Micrograph of sample from $(\mathbf{C})$. Bar $=100 \mu \mathrm{m}$. Note the marked binding of antifilaggrin to the parakeratinized superficial layers of the epithelium. E. Micrograph of conjunctiva with moderate dysplasia. Bar $=100 \mu \mathrm{m}$. Only focal filaggrin expression was found. F. Micrograph of normal conjunctiva, which does not express filaggrin. Bar $=100 \mu \mathrm{m}$. G. Micrograph of normal skin from same patient as in $(\mathbf{F})$. Bar $=100 \mu \mathrm{m}$. Note the marked expression of filaggrin in the keratinized layers of the epithelium. 
mutation. Other more sensitive methods of measuring filaggrin in the conjunctiva could also be used to confirm the results.

In conclusion, filaggrin is not a sensitive marker of mechanical stress to the conjunctiva. However, our results show that filaggrin is expressed in Stevens-Johnson syndrome with severe parakeratinization. Filaggrin may also be expressed in conjunctival dysplasia.

\section{CONFLICT OF INTEREST}

The authors confirm that this article content has no conflict of interest.

\section{ACKNOWLEDGEMENTS}

This study was supported by Lundbeck Foundation, Copenhagen.

\section{REFERENCES}

[1] De Benedetto A, Qualia CM, Baroody FM, Beck LA. Filaggrin expression in oral, nasal, and esophageal mucosa. J Invest Dermatol. 2008;128: 1594-7.

[2] Cintorino M, Syrjanen S, Leoncini P, et al. Altered expression of filaggrin in human papillomavirus (HPV) lesions of the uterine cervix. Arch Gynecol Obstet 1988; 241: 235-47.

[3] Tong L, Corrales RM, Chen Z, et al. Expression and regulation of cornified envelope proteins in human corneal epithelium. Invest Ophthalmol Vis Sci 2006; 47: 1938-46.
[4] Saika S, Minamide A, Tanaka T, et al. Expression of involucrin by ocular surface epithelia of patients with benign and malignant disorders. Curr Eye Res 2000; 21: 877-85.

[5] Krenzer KL, Freddo TF. Cytokeratin expression in normal human bulbar conjunctiva obtained by impression cytology. Invest Ophthalmol Vis Sci 1997; 38: 142-52.

[6] Nakamura T, Nishida K, Dota A, Matsuki M, Yamanishi K, Kinoshita S. Elevated expression of transglutaminase 1 and keratinization-related proteins in conjunctiva in severe ocular surface disease. Invest Ophthalmol Vis Sci 2001; 42: 549-56.

[7] Spencer WH. Ophthalmic pathology, an atlas and textbook. 3. ed. Philadelphia: W.B. Saunders 1985.

[8] Sandilands A, Sutherland C, Irvine AD, McLean WH. Filaggrin in the frontline: role in skin barrier function and disease. J Cell Sci 2009; 122: 1285-94.

[9] O'Regan GM, Sandilands A, McLean WH, Irvine AD. Filaggrin in atopic dermatitis. J Allergy Clin Immunol 2009; 124(3 Suppl 2): R2-6.

[10] Dale BA, Holbrook KA, Kimball JR, Hoff M, Sun TT. Expression of epidermal keratins and filaggrin during human fetal skin development. J Cell Biol 1985; 101: 1257-69.

[11] Smith FJ, Irvine AD, Terron-Kwiatkowski A, et al. Loss-offunction mutations in the gene encoding filaggrin cause ichthyosis vulgaris. Nat Genet 2006; 38: 337-42.

[12] Sandilands A, O'Regan GM, Liao H, et al. Prevalent and rare mutations in the gene encoding filaggrin cause ichthyosis vulgaris and predispose individuals to atopic dermatitis. J Invest Dermatol 2006; 126: 1770-5.

[13] Palmer CN, Irvine AD, Terron-Kwiatkowski A, et al. Common loss-of-function variants of the epidermal barrier protein filaggrin are a major predisposing factor for atopic dermatitis. Nat Genet 2006; 38: 441-6. 